1998, Vol. 8, Nos. (1-4), pp. $117-12$

Reprints available directly from the publisher

Photocopying permitted by license only
(C) 1998 OPA (Overseas Publishers Association) N.V.

Published by license under the Gordon and Breach Science Publishers imprint. Printed in India.

\title{
X3D Moving Grid Methods for Semiconductor Applications*
}

\author{
ANDREW KUPRAT ${ }^{\dagger}$, DAVID CARTWRIGHT, J. TINKA GAMMEL, \\ DENISE GEORGE, BRIAN KENDRICK, DAVID KILCREASE, \\ HAROLD TREASE and ROBERT WALKER
}

Los Alamos National Laboratory

\begin{abstract}
The Los Alamos 3D grid toolbox handles grid maintenance chores and provides access to a sophisticated set of optimization algorithms for unstructured grids. The application of these tools to semiconductor problems is illustrated in three examples: grain growth, topographic deposition and electrostatics. These examples demonstrate adaptive smoothing, front tracking, and automatic, adaptive refinement/derefinement.
\end{abstract}

Keywords: Adaptive mesh smoothing, multimaterial grids, moving grids, moving surfaces, unstructured grids, moving finite elements

\section{INTRODUCTION}

3D grain growth modeling and 3D topographic simulation have in common the requirement of accurately representing time dependent surface motion in a $3 \mathrm{D}$ volume. Problems involving fixed surfaces such as parasitic parameter extraction for interconnect modeling can benefit from adaptive grid methods because they substantially reduce solution error from iteration to iteration. The Los Alamos X3D grid toolbox provides a set of capabilities including initial grid generation of complex multimaterial geometries and grid optimization that preserves material interfaces. X3D data structures and toolbox methods are designed as objects in order to be user accessible and extensible. X3D commands can be issued from within an application driver program, and the example applications use this feature to perform, as needed, grid reconnection, node merging and smoothing. Additionally, all X3D data structures are available to the application driver via calls to utility routines. Thus when an application detects a non-routine event such as a topological change in a material region under deformation, a special purpose user routine can easily be incorporated into the system. These design features promote the separation of the physical based simulation from

*Work supported by the U.S. Department of Energy.

${ }^{\dagger}$ Corresponding author. 
the grid maintenance chores, but allow full access to the grid data structures when required.

\section{Gradient Weighted Moving Finite Elements Applied to Metallic Grain Growth}

In our first application involving the X3D toolbox [1], we use Gradient Weighted Moving Finite Elements [2] (GWMFE) to move a multiplyconnected network of triangles to model the annealing of 3-D metallic grains. We assume evolution of grain interfaces obeys the simple equation

$$
v_{\mathrm{n}}=\mu K,
$$

where $v_{\mathrm{n}}$ is the normal velocity of the interface, $\mu$ is the mobility, and $K$ is the local mean curvature [3]. Gradient Weighted Moving Finite Elements minimizes

$$
\int\left(v_{\mathrm{n}}-\mu K\right)^{2} d S
$$

over all possible velocities of the interface vertices. (The integral is over the surface area of the interfaces.) This leads to system of $3 N$ ODE's:

$$
\mathbf{C}(\mathbf{y}) \dot{\mathbf{y}}=\mathbf{g}(\mathbf{y}),
$$

where $\mathbf{y}$ is the $3 N$-vector containing the $x, y$, and $z$ coordinates of all $N$ interface vertices, $\mathbf{C}(\mathbf{y})$ is the matrix of inner products of finite element basis functions, and $\mathbf{g}(\mathbf{y})$ is the right-hand side of inner products involving surface curvature. The ODE's are integrated using an implicit variable time step integrator.

As an example, we evolve a 5 grain microstructure in the confined geometry of an aluminum interconnect on a semiconductor chip, and in Figure 1 we show the smooth surfaces of the grains at an intermediate time in their evolution under mean curvature. (The initial state for the time evolution was obtained using Monte Carlo annealing of a discrete effective model on a fixed lattice [4] and is not shown.) Visible in this exploded view

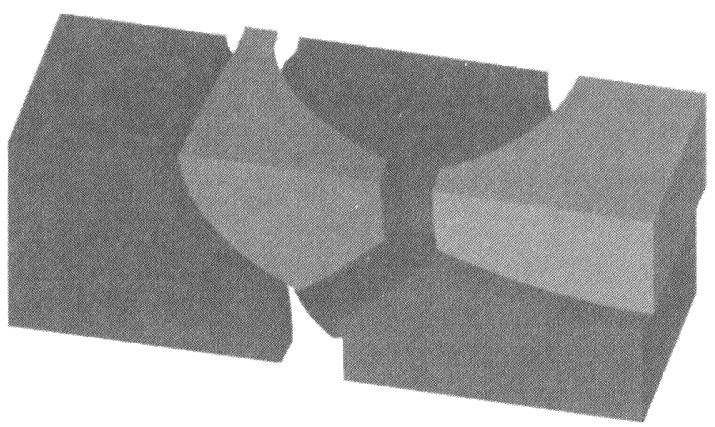

FIGURE 15 grain microstructure evolved under mean curvature motion using GWMFE.

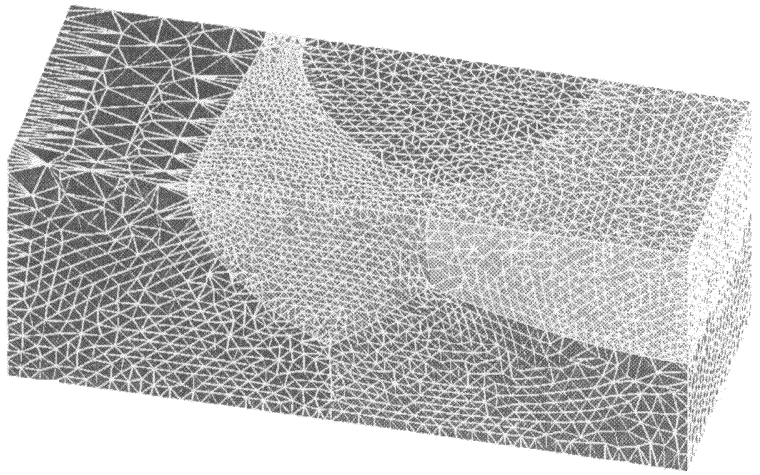

FIGURE 2 Evolved 5 grain microstructure showing surface grid. Evolution has caused imbalance in grid density.

are triple points on the surface of the interconnect, as well as a triple line and tetrahedral point in the interior. The corresponding surface grid is shown in Figure 2. It is clear that for this simulation to continue, some of triangles must be annihilated to prepare for topological events such as the "pinching off" of a grain. That is, a front tracking simulation must involve a nontrivial amount of grid manipulation in order to successfully run to completion. In Figure 3, we show the effect of massage which is a grid manipulation command in the X3D toolbox. As seen in the figure, the massage command has derefined the unstructured mesh without significantly damaging the shapes of the grains. Indeed, the command takes as user input a "damage" tolerance which gives the maximum acceptable amount of grain shape deformation allowable in the derefinement pro- 


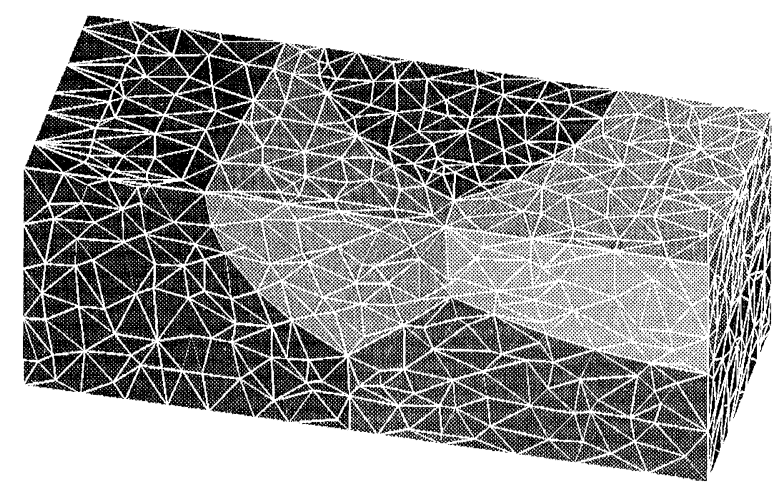

FIGURE 35 grain evolved grid after X3D massage command. The grid has been derefined and refined according to desired edge length criteria while preserving material interfaces and external boundaries.

cess. Also called is the recon command which allows changing of connectivity of the associated tetrahedral mesh in the interior of the volume. Still a work in progress, we anticipate that with the availability of these X3D grid manipulation tools we will be able to run the annealing process to steady-state. These microstructures will then provide high quality three-dimensional models for electromigration reliability simulation.

\section{Finite Volume Electrostatic Calculations on a Solution Adapted Mesh}

Problems involving fixed surfaces can also benefit from adaptive grids. To illustrate this, we show the effects of solution adaptive grid generation for calculation of electric fields in nontrivial geometry - as occurs when attempting to extract parasitic parameters for interconnect modeling. Figure 4 shows the interior of a box with a conical intrusion. We solve Laplace's equation on this three-dimensional domain using a finite volume solver. (Only the surface triangles are shown in the figure; the calculation is done on unshown tetrahedra that conform to the surfaces and fill the volume.) As is well known, the electric field becomes arbitrarily large near the tip of the cone. Displayed at the tip of the cone is an isosurface for the component of electric field aligned with the

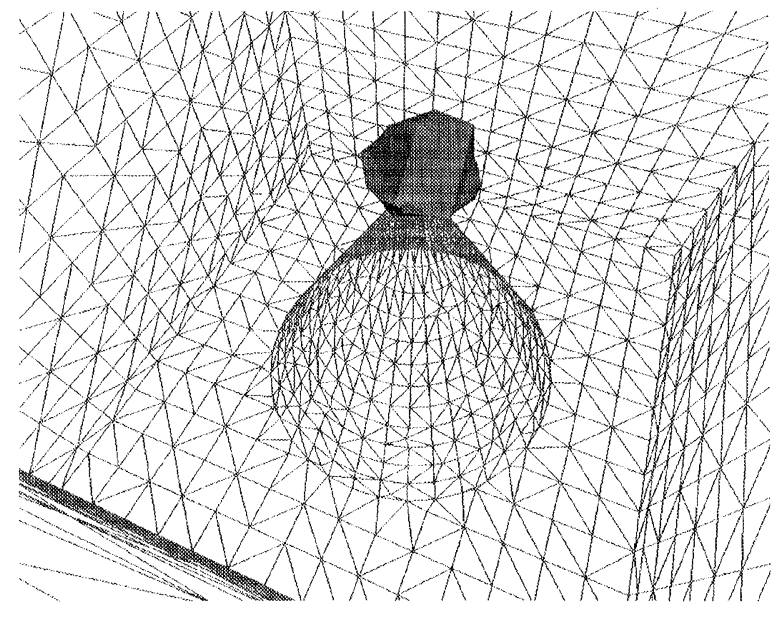

FIGURE 4 Surface triangles from an unadapted grid used to solve Laplace's equation on a unit cube with the bottom surface pierced by a sharp cone. Displayed at the tip of the cone is an isosurface of the component of the electric field aligned with the axis of the cone.

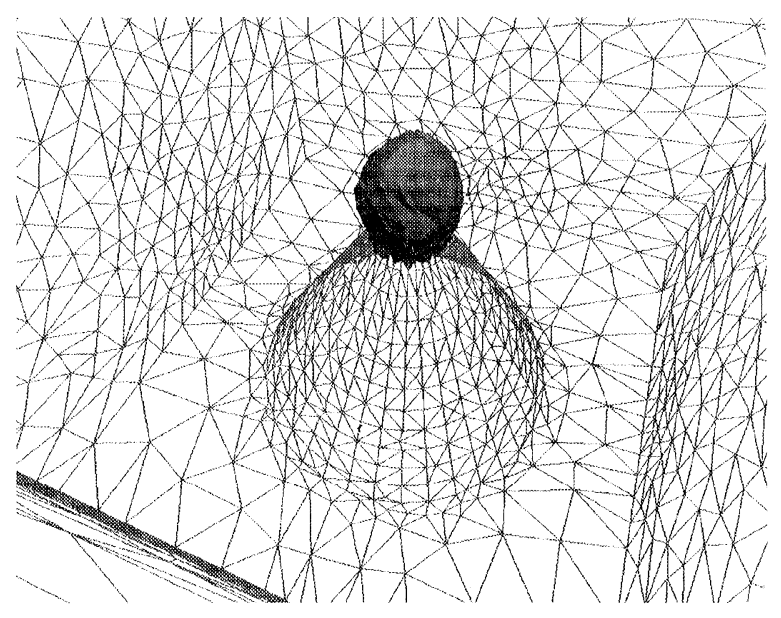

FIGURE 5 Surface triangles after error-dependent grid adaption. Note improvement in electric field isosurface. Adaption was turned off on the surface of the cone to prevent too much grid from disappearing into the tip of the cone where the electric field is infinite.

axis of the cone. As can be seen, this isosurface is nonsmooth due to lack of resolution in this area of highest solution error. In Figure 5 we show the same view and electric field component isosurface for the solution on a grid that has been adapted to an a posteriori error estimate. The isosurface is smoother, indicating that adaptive error-based 
smoothing has successfully attracted the solution elements to the critical area near the tip of the cone. (However, to prevent the entire mesh from disappearing into the singularity at the tip of the cone, node movement on the surface of the cone was turned off.)

The solution adaption was accomplished using the X3D smooth command which adapts a mesh to minimize the $\mathcal{L}^{2}$ norm of the gradient of solution error, as calculated using an a posteriori error estimate based on estimated second derivatives of the solution [5]. In order for the finite volume method to work, a Delaunay grid is required. Thus, after each adaptive smoothing iteration, the $\mathrm{X} 3 \mathrm{D}$ recon command is called to adjust the mesh connectivity to restore the Delaunay condition on the tetrahedral mesh. We note that the maximum electric field on the adapted mesh of Figure 5 is approximately ten times higher than that on the unadapted mesh of Figure 4. Thus, without increasing the number of nodes used, the solution adaption using X3D toolbox commands was automatically able to increase the quality of the solution in a critical area.

\section{D Topographic Simulation}

In simulating topographic etch and deposition, TopoSim3D separates the chemical and physical processes from grid generation and maintenance operations. The flux calculation, source characterization and chemical reaction mechanisms have access to the needed X3D data structures and to the X3D geometry services. X3D is used to build the initial 3D tetrahedral mesh that represents the wafer and to accurately track the evolution of the material interfaces as they change with time. As material is deposited or etched away from interfaces, those tetrahedral faces (interface triangles) that lie on the boundary between two materials move in time. From a computation of the flux of materials arriving at the surface of the interface triangles, we move triangle nodes after apportioning the area of each triangle to each of the nodes it shares. The velocity of each node is then computed from an area-weighted vector average of the contributions from each of the triangles that share the node.

In low pressure simulations, the transport of material between the boundary plane and the surface of a wafer is represented by straight-line trajectories of particles. The flux of materials arriving at a triangle on the surface interface depends on the source emanation rate, the distance between the source and the surface element, the relative orientation of the source and the surface element, and the visibility of the surface as viewed from the source. In the simplest model, all the material arriving at the wafer surface stays there, implying that the only source elements are those that lie on the boundary plane. However, if some vapor phase materials arriving at the surface do not react there, they (or other species) will be reemitted, and in such cases, all interface triangles serve as potential source elements. By specifying the surface chemistry (using the ChemKin and Surface ChemKin reaction software libraries), TopoSim3D incorporates the calculation of "sticking coefficients" in a natural way. If the rate of a surface reaction is slower on a surface element

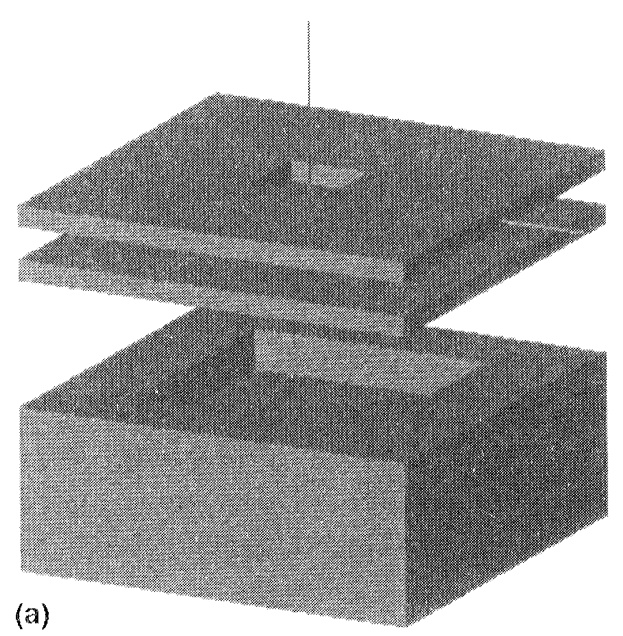

FIGURE 6 Topographic deposition onto two materials with different 'sticking coefficients'. View $a$ shows the solid model of the wafer. View $b$ gives the initial surface grid of the hole with the front surface of the hole removed. View $c$ shows an intermediate time step in the deposition. View $d$ shows the final time step in the deposition. Note the differences in upper and lower material 'sticking coefficients'. 

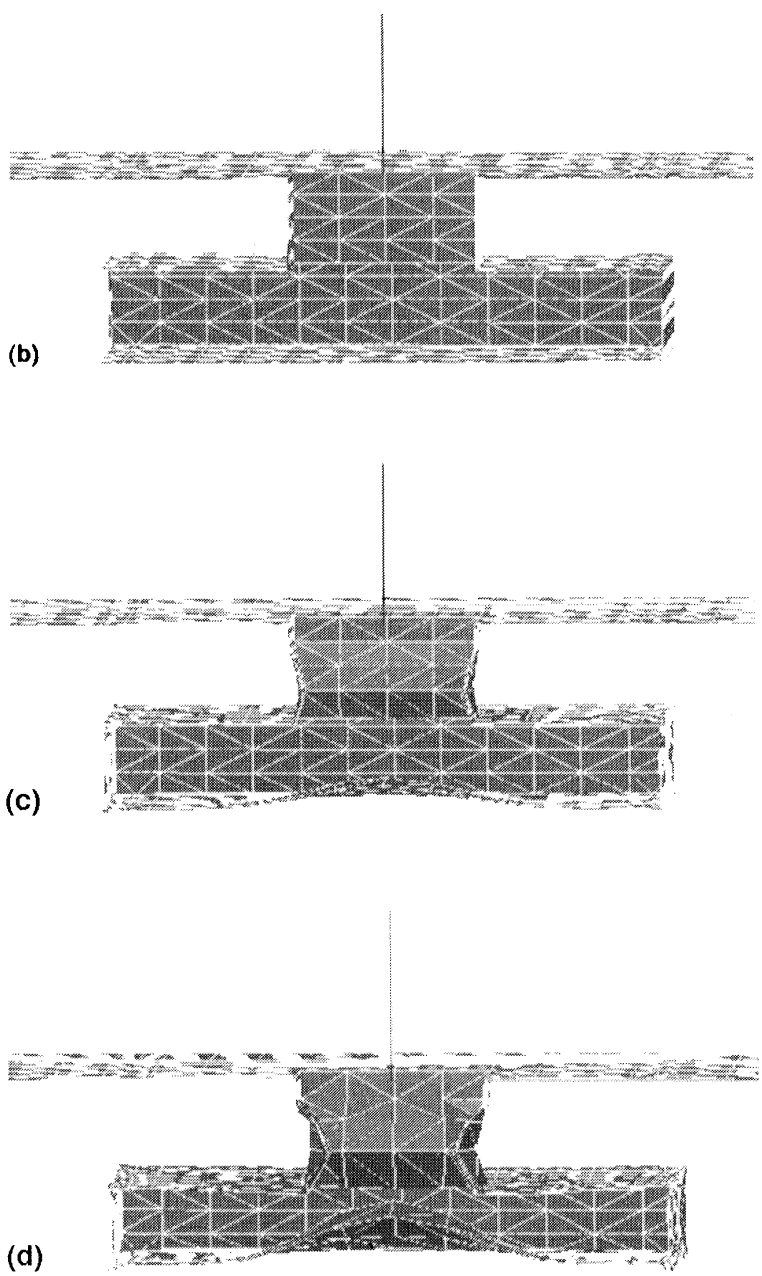

FIGURE 6 (Continued).

than the arrival rate of reactants, mass balance requires the excess material to be re-emitted from that interface triangle, which in turn becomes an effective source element for other surface elements.

As in grain growth modeling, the $\mathrm{X} 3 \mathrm{D}$ grid maintenance calls are used to refine, reconnect and smooth the interface surface during the time evolution. TopoSim3D uses the X3D data struc- tures to help avoid folding problems and detect topological events such as a pinch-off. Figure 6 shows deposition on an overhang structure with material-dependent sticking coefficients.

\section{CONCLUSION}

As shown in the three examples presented, X3D grid optimization techniques are well suited to solving time-dependent and geometrically challenging problems occurring in semiconductor applications.

\section{References}

[1] George, D. C. (1995). X3D User's Manual, Los Alamos National Lab Report, LA-UR-95-3608.

[2] Miller, K. (1997). A Geometrical-Mechanical Interpretation of Gradient-Weighted Moving Finite Elements, SIAM J. Num. Anal., 34, 67-90.

[3] Porter, D. A. and Easterling, K. E. (1988). Phase Transformations in Metals and Alloys, Great Britain: Van Nostrand Reinhold, pp. 130-136.

[4] Kuprat, A. and Gammel, J. T. Modeling Metallic Microstructure Using Moving Finite Elements, preprint, available at http://xxx.lanl.gov/abs/physics/9705041.

[5] Bank, R. E. and Smith, R. K. (1997). Mesh smoothing using a posteriori error estimates, SIAM J. Num. Anal., 34, 979-997.

\section{Author Biography}

Andrew P. Kuprat received his Ph.D. in Mathematics from the University of California at Berkeley and is currently a staff member at Los Alamos National Laboratory. He is interested in adaptive mesh algorithms applied to problems in semiconductor process and device modeling, as well as to other convection/diffusion problems in physics. He is also interested in mesh generation and computational geometry. 

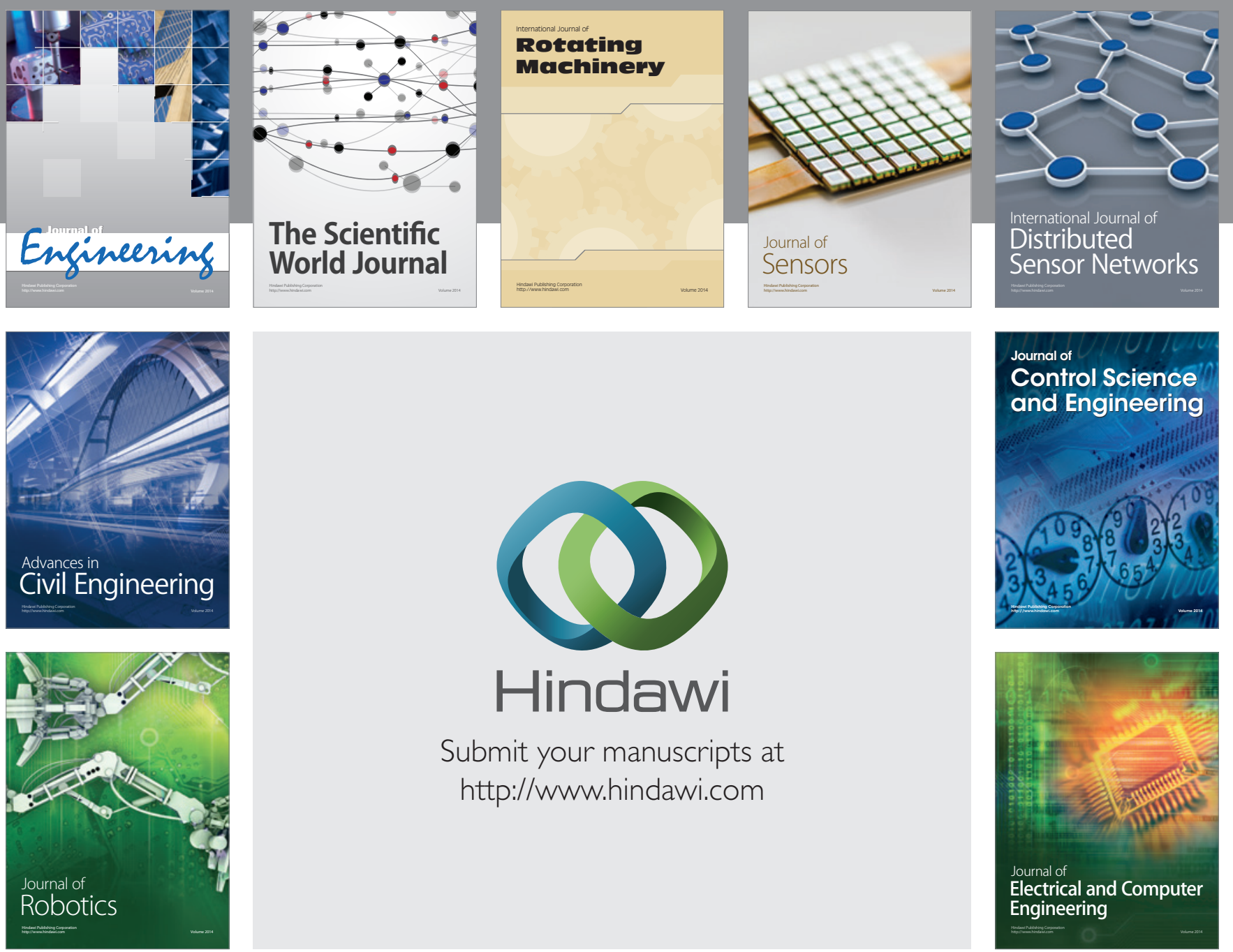

Submit your manuscripts at

http://www.hindawi.com
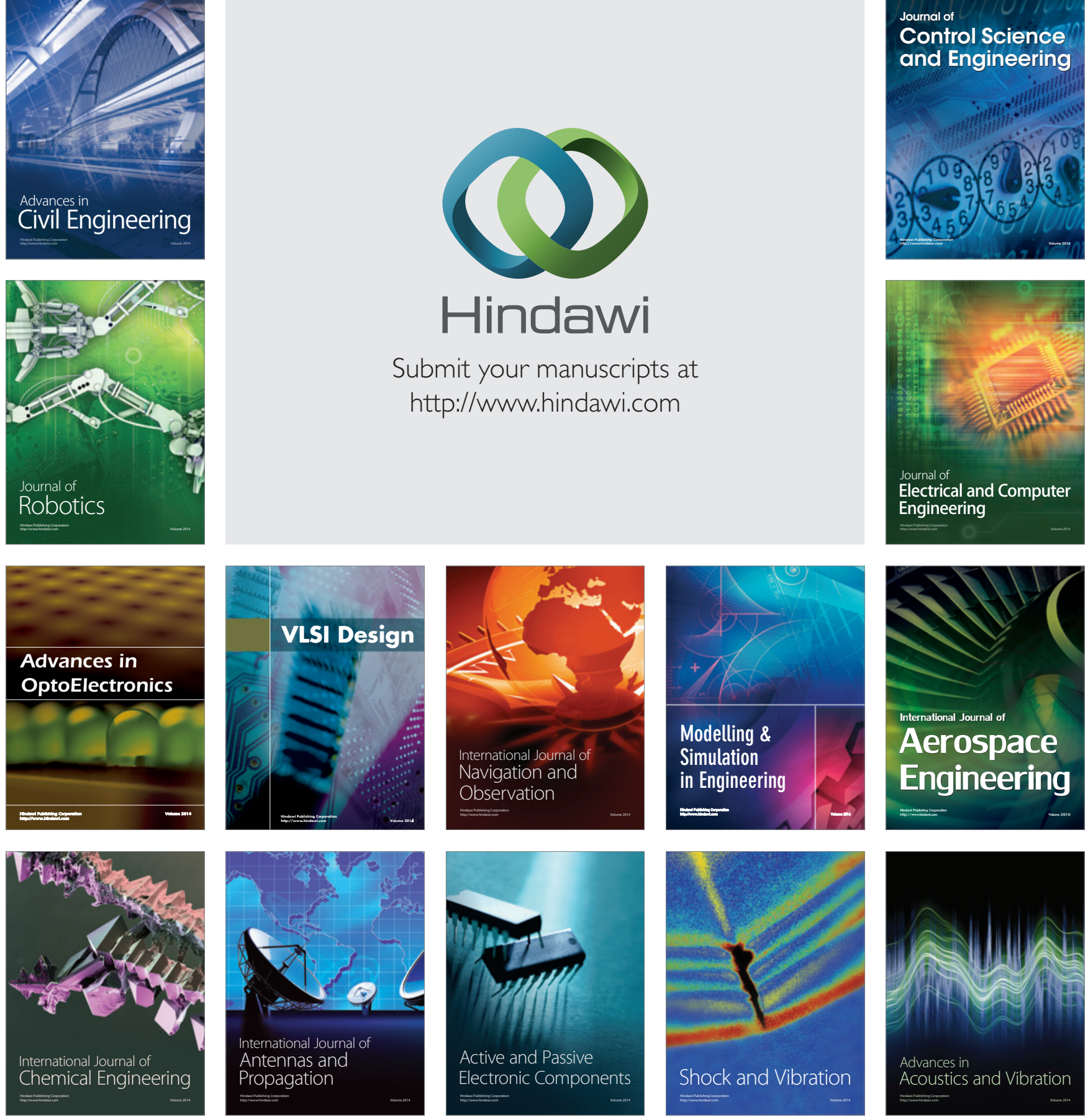